\title{
Infecciones de transmisión sexual silentes: la muestra de orina permite una adecuada detección
}

\author{
Javier Retamal, Raúl Sánchez y Priscilla Brebi
}

\section{Non symptomatic sexually transmitted infections: urine sample allows an adequate detection}

Detection of non-symptomatic sexually transmitted infections (NSSTD) has taken great relevance, primarily due to global increase. This has led to implement various laboratory techniques with the aim of early detection of these silent infections to decrease the incidence. Techniques usually used for the detection and identification of NSSTD require invasive samples (blood, citobrush, etc.), so the urine could be a simpler option and noninvasive sample when the patient be subjected to test for some of these infections.

Key words: Non-symptomatic sexually transmitted infections, urine sample, noninvasive sample, nucleic acid amplification techniques, Mycoplasma spp., Ureaplasma spp., Chlamydia trachomatis, human papillomavirus, herpes simplex virus, human immunodeficiency virus.

Palabras clave: Infecciones de transmisión sexual silentes, muestra de orina, muestra no invasora, técnicas de amplificación de ácidos nucleicos, Mycoplasma spp., Ureaplasma spp., Chlamydia trachomatis, virus papiloma humano, virus herpes simplex, virus de la inmunodeficiencia humana.

\section{Introducción}

L as infecciones de transmisión sexual (ITS) son un grupo heterogéneo de infecciones que pueden afectar tanto a hombres como mujeres y cuyo único elemento en común es compartir la vía sexual como mecanismo de transmisión ${ }^{1}$.

Las ITS más comunes y que representan un importante problema de salud pública son provocadas por microorganismos tales como Neisseria gonorrhoeae (gonorrea), Chlamydia trachomatis (clamidia), Mycoplasma genitalium (micoplasma), virus de la inmunodeficiencia humana (VIH), virus papiloma humano (VPH) y virus herpes simplex (VHS). Las infecciones de origen bacteriano por lo general son tratables y curables a la vez, no así las de origen viral, que si bien son tratables, no todas pueden ser eliminadas del organismo.

La Organización Mundial de la Salud estima que cada año se producen aproximadamente 498,9 millones de nuevos casos de ITS en el mundo, curables, en hombres y mujeres adultas de 15 a 49 años, cifra que no incluye las infecciones incurables, entre ellas infecciones por $\mathrm{VIH}^{2}$.

Las ITS pueden ser sintomáticas o asintomáticas (silentes). Las infecciones sintomáticas más comunes son las provocadas por bacterias como $N$. gonorrhoeae cuya incidencia global por año es de 106,1 millones de casos²; la incidencia en Chile fue de 1.428 casos reportados en el año $2011^{3}$.
Estas infecciones también pueden encontrarse en el organismo de manera asintomática ${ }^{4,5}$ dificultando su detección. Las ITS silentes (ITSS) más frecuentes universalmente son provocadas por el VPH $(2-44 \%)^{6}, C$. trachomatis $^{7}$ y VHS tipo II $(16,2 \%)^{8}$.

Las ITS silentes han producido un elevado número de pacientes infectados sin que éstos estén en conocimiento de su condición. Se detectan en general cuando los pacientes presentan algún problema de salud, por ejemplo en la mujer un PAP alterado o infertilidad. Este tipo de infecciones representan uno de los principales problemas de salud relacionados con la función reproductiva y sexual, constituyendo una de las principales causas de infertilidad, debido a infecciones causadas por microorganismos no tratados ${ }^{9}$. Entre otras complicaciones asociadas a las ITSS se encuentra el desarrollo de cáncer (cérvix, pene, región perianal, piel, amígdala, orofaríngeo, laríngeo, esófago, próstata y uretra $)^{10}$, enfermedad inflamatoria pélvica, embarazos ectópicos ${ }^{11}$ y enfermedades asociadas a inmunodepresión como la hepatitis $\mathrm{B}^{12,13}$.

Debido a la alta incidencia, morbilidad y mortalidad que ocasionan y los costos asociados, las ITSS son consideradas enfermedades de alta prioridad en su detección en el mundo, por lo que es trascendental desarrollar métodos de detección sensibles y específicos que faciliten disminuir la transmisión y las secuelas provocadas por estas infecciones ${ }^{14}$.
Universidad de La Frontera.

Temuco, Chile.

Facultad de Medicina, BIOREN-CEGIN, Departamento de Patología, Laboratorio de Patología Molecular (JR, PB)

Departamento de Ciencias Preclínicas, Laboratorio de Virus Oncológicos (RS).

Los autores no poseen conflictos de intereses para la publicación de este artículo.

Esta investigación fue financiada por el Proyecto CORFO NN 12IDL218157, Proyecto DIUFRO DI13-0039 y Proyecto CORFO-CEGIN No 09CN14-5960.

Recibido: 24 de noviembre de 2014 Aceptado: 17 de abril de 2015

Correspondencia a: Priscilla Brebi Mieville brebimieville@gmail.com 


\section{Detección de ITSS}

El diagnóstico de las ITSS ha ido evolucionado muy rápidamente debido a las dificultades presentes al tomar muestras por procedimientos invasores y especialmente por la baja cantidad de antígenos y de copias del microorganismo presentes en la muestra. Esto dificulta la detección y diagnóstico por medio de métodos tradicionales tales como inmunofluorescencia, enzimoinmunoanálisis, o sondas de $\mathrm{ADN}^{15}$. En la actualidad es posible detectar y diagnosticar las ITSS no sólo mediante cultivos microbiológicos sino que también por técnicas inmunológicas y moleculares.

\section{Técnicas utilizadas en la detección de ITSS}

El cultivo ha sido utilizado como referencia en el diagnóstico de las ITS, debido a su sensibilidad, especificidad y bajo costo. Los resultados obtenidos mediante esta técnica están estrechamente relacionados con el tipo de muestra utilizada y el correcto manejo y transporte de la misma. Sin embargo, para entregar un diagnóstico definitivo es necesaria la realización de pruebas bioquímicas o técnicas inmunológicas ${ }^{16}$. Durante la década de los 80's aparecieron nuevas técnicas de diagnóstico, que se destacaron por no requerir del cultivo, como la inmunofluorecencia, el enzimoinmunoanálisis (EIA) y la hibridación con sondas de ADN. Estas técnicas requieren un alto número de microorganismos (entre $10^{4}$ y $10^{7}$ ) por lo que su sensibilidad es aproximadamente de $70 \%{ }^{17}$.

En la década de los 90's aparecieron las técnicas de amplificación de ácidos nucleicos (TAAN), como la reacción de polimerasa en cadena $(\mathrm{RPC})^{18}$, reacción de la ligasa en cadena (LCR en inglés) ${ }^{19}$ y la técnica de amplificación mediada por transcriptasa (TMA en inglés $)^{20}$. A diferencia de los métodos de cultivo y técnicas inmunológicas, las TAAN son capaces de detectar un bajo número de microorganismos (entre 10 y $10^{2}$ ), logrando alcanzar una especificidad de $95 \%$ y una sensibilidad de $90-95 \%$. Esto posibilita la detección de un mayor número de infecciones en comparación con los métodos de cultivo y técnicas inmunológicas ${ }^{15}$.

En la actualidad, es posible detectar la presencia de Mycoplasma spp. y Ureaplasma spp. mediante la utilización de TAAN, como la RPC-TR, que permite una rápida detección de estos microorganismos con una elevada sensibilidad y especificidad ${ }^{21-23}$, en comparación a la técnica de cultivo. El cultivo, a pesar de ser señalado como la técnica más confiable y de primera elección, es considerablemente lenta (alrededor de 6 días), además es sensible a cambios de $\mathrm{pH}$ y presencia de otras bacterias ${ }^{24}$. Otro método de diagnóstico es la utilización de sistemas API ${ }^{\circledR}$, que emplea una batería de pruebas bioquímicas dispuestas en una placa plástica con microtubos, que contienen anticuerpos, sustratos de enzimas y medios de cultivo deshidratados específicos para estos microorganismos ${ }^{25}$.

Para la detección de C. trachomatis, la técnica de cultivo en líneas celulares fue el referente para el diagnóstico por muchos años, debido a su especificidad, que alcanza aproximadamente a $100 \%$, pero con una baja sensibilidad $(70-85 \%)^{26-28}$. En el año 2002, junto con las mejoras a las TAAN y la obtención de muestras menos invasoras, como lo es la orina, se generaron mejores oportunidades para lograr un diagnóstico más rápido y preciso de $C$. trachomatis. Actualmente, la FDA recomienda el uso de TAAN que han logrado alcanzar una sensibilidad de $93,8 \%$ y especificidad de $100 \%{ }^{29,30}$; con ellas se ha logrado detectar de 20 a $50 \%$ más infecciones que con la técnica de cultivo ${ }^{31}$.

Estas nuevas TAAN también son utilizadas en la detección de $N$. gonorrhoeae; se han desarrollado test basados en RPC obteniéndose resultados con altos valores de especificidad, sensibilidad y una considerable disminución en el tiempo de detección en comparación a las técnicas tradicionales de cultivo y test de susceptibilidad antimicrobiana $^{32}$.

El VPH no puede ser cultivado con métodos tradicionales de cultivo celular y las técnicas serológicas tienen una precisión limitada, debido a que a raíz de la infección se produce una respuesta inmune humoral contra las proteínas de cápside mayor con anticuerpos que persisten en el tiempo; entonces no es posible distinguir entre una infección reciente y una infección pasada mediante serología ${ }^{33}$. Por esto el diagnóstico de VPH es realizado mediante la detección de ADN viral ${ }^{34}$. Los métodos moleculares para la detección de ADN de VPH se pueden agrupar principalmente en dos categorías: aquellos que se basan en la amplificación de señal para detectar el ADN viral, y los que se basan en la amplificación de dichos ácidos nucleicos. La mayoría de ellos se basa en la RPC siendo el método de amplificación más usado. El Cobas ${ }^{\circledR} 4800$ (Roche Diagnostics, Indianapolis, IN, USA) es un sistema automatizado de RPC-TR con el que es posible detectar por separado los genotipos $16 \mathrm{y}$ 18 , además de otros genotipos que encierran alto riesgo oncogénico $^{35}$. Otro método utilizado, es el de captura híbrida, considerado como el método más confiable en la detección de 13 genotipos de alto riesgo; sin embargo, éste no permite diferenciar el tipo específico de VPH y discriminar infecciones múltiples ${ }^{36,37}$.

La infección provocada por el VHS puede ser detectada en la actualidad mediante TAAN como la RPC-TR ${ }^{38,39}$. Esta técnica permite diferenciar en un solo test infecciones provocadas por VHS-1 y VHS-2, cuyos genotipos presentan una gran similitud ${ }^{40}$ y a su vez disminuir el tiempo de detección y la cantidad de muestra utilizada considerablemente. La técnica de RPC es la más adecuada en la 
detección de VHS ${ }^{41}$ alcanzando valores de sensibilidad y especificidad cercanos a $95 \%{ }^{42}$. Se ha demostrado que la técnica de RPC presenta una mayor sensibilidad por sobre la técnica de cultivo viral, actualmente considerada el estándar de oro en la detección de $\mathrm{VHS}^{43}$.

Las técnicas de detección de la infección por VIH determinan la presencia de anticuerpos en el suero prácticamente en $100 \%$ de las personas infectadas con una sensibilidad y especificidad alrededor de $99 \%$, sin embargo, el 1\% restante puede influenciar en la obtención de falsos negativos en el caso de la sensibilidad y falsos positivos en el caso de la especificidad, por lo que se recomienda la utilización de un test confirmatorio ${ }^{44}$. La técnica de ELISA utilizada en el tamizaje de VIH, ha experimentado numerosos cambios, ya que en el año 1985 se utilizaba lisado vírico como base antigénica, logrando detectar los anticuerpos 40 días después de la infección ${ }^{45}$. En el año 1987 el lisado fue sustituido por proteínas recombinantes y péptidos sintéticos, aumentando la sensibilidad y detectando la presencia de anticuerpos 33-35 días luego de la infección ${ }^{46}$. En el año 1994 la técnica ELISA adquirió el formato sandwich, logrando detectar anticuerpos IgG e IgM y reducir el período de detección a 22 días $^{46}$. Actualmente, se utilizan técnicas que posibilitan la detección simultánea de anticuerpo y el antígeno p24, reduciendo el período de detección a aproximadamente 13-15 días, alcanzando un porcentaje de sensibilidad de $99,9 \%{ }^{45}$.

\section{Tipos de muestras clínicas utilizadas en la detección de ITSS}

Cualquier tipo de muestra clínica podría tener el potencial para ser utilizada en el diagnóstico de una ITS silente. No obstante, en procedimientos rutinarios se utiliza un número reducido de tipos de muestras clínicas procurando manipularlas con cautela y guiándose por los protocolos establecidos ${ }^{14}$.

Las técnicas de detección de ITSS mencionadas anteriormente utilizan muestras clínicas invasoras como suero sanguíneo y exudados de tipo uretral, endocervical, vaginal, anal, faríngeo, úlceras, y muestras no invasoras como la orina.

Las muestras de suero son utilizadas en el diagnóstico de ITSS mediante el uso de técnicas serológicas, como es el caso de C. trachomatis ${ }^{47}$ y del $\mathrm{VIH}^{45}$, técnicas que tienen como finalidad encontrar anticuerpos desarrollados por el organismo en respuesta a la infección ${ }^{48}$.

Los exudados son las muestras clínicas preferidas para la detección de infecciones de ITSS. Se recolectan utilizando tórulas de alginato cálcico o dacrón y se utiliza un medio de transporte tipo Stuart-Amies ${ }^{16,49}$. Lo ideal es utilizar varias tórulas consecutivas para procurar ob- tener la mayor cantidad de exudado. Sus ventajas son su bajo costo, no producen traumatismo y provocan escasa disconformidad; sin embargo, las células pueden quedar capturadas en sus fibras. Otra técnica de recolección es la aspiración y raspado de la lesión utilizando un escalpelo en el lugar donde se tomará la muestra clínica ${ }^{16,49}$.

Actualmente, para la toma de muestras endocervicales se utiliza un citobrush, el cual consiste en un cepillo de nylon con cerdas perpendiculares al mango. Dentro de sus ventajas, esta que sus fibras maleables de nylon permiten la inserción en el canal para una mejor toma de muestra, y evita que las células queden atrapadas en ellas, y por último, sus fibras rígidas pueden penetrar glándulas. No obstante, estas fibras rígidas pueden provocar traumatismos y causar dolor ${ }^{50}$.

La orina como muestra clínica, fue una de las primeras utilizadas por el hombre en la antigüedad y su uso en exámenes data de la época de los egipcios. Mediante pruebas fisicoquímicas es posible diagnosticar diversas patologías renales y pre renales, dejando al descubierto afecciones del tracto urinario, hepatopatías, enfermedades hemolíticas y trastornos en el metabolismo de los carbohidratos entre otros ${ }^{51}$. La muestra de orina para urocultivo es la más frecuentemente recibida en laboratorios de microbiología, permitiendo detectar diversos microorganismos causantes de infecciones urinarias o bacteriurias asintomáticas ${ }^{52,53}$.

La orina es una la única muestra clínica no invasora en el diagnóstico y detección de ITSS y es usada principalmente para técnicas moleculares, que poseen una mayor sensibilidad y especificidad en comparación a las técnicas tradicionales. Además, el paciente la puede tomar privadamente y sin riesgo de equivocación en la obtención. Los resultados obtenidos mediante el uso de este tipo de muestra son comparables al uso de exudados uretrales y cervicales ${ }^{54}$.

\section{Detección de ITSS en orina}

El uso de orina en el diagnóstico de Mycoplasma genitalium mediante la utilización de la técnica de RPC, tiene una sensibilidad de $\sim 60 \%$, en comparación con muestras como exudado vaginal $(85,7 \%)$ y exudado endocervical $(74,3 \%)^{55}$. Esta muestra puede ser utilizada tanto para cultivo como para detección a través de técnicas de biología molecular, permitiendo disminuir los falsos negativos de la serología y aumentar la pesquisa de pacientes que presenten este tipo de infecciones ${ }^{56}$.

Como se mencionó anteriormente, la amplificación de ácidos nucleicos es la técnica sensible con mayor especificidad para la detección de la infección por $C$. trachomatis, la que se realiza principalmente utilizando muestras tales como, citobrush, secreción vaginal y/o secreción uretral ${ }^{29}$. Nuevas evidencias concluyen que la 
muestra de orina también sería adecuada para la detección de este microorganismo, ya que igualmente puede alojarse y multiplicarse en la uretra y producir uretritis no gonocóccica, tanto en hombres como en mujeres. Por ello, este tipo de muestra, sería de gran utilidad para detectar C. trachomatis en ambos géneros ${ }^{56}$. En el caso de la detección en mujeres, en necesario complementar la muestra de orina junto con citrobrush, ya que la infección por $C$. trachomatis puede afectar tanto el tracto genital como el tracto uretral por separado ${ }^{57}$.

Según el Centro de Control y Prevención de Enfermedades (CDC por sus siglas en inglés), la muestra de orina es una de las muestras utilizadas actualmente en la detección de infecciones causadas por $N$. gonorrhoeae. Acompañada de TAAN logra alcanzar valores de sensibilidad y especificidad de 95 y $>99 \%$, respectivamente ${ }^{31}$. En el caso del VHS no se encontraron valores de sensibilidad y especificidad en muestras de orina.

Las muestras de orina son ampliamente utilizadas en la detección de VPH debido al alto nivel de aceptación que tiene este tipo de muestra en la población y además por su fácil obtención ${ }^{58}$. Lamentablemente, la cantidad de ADN viral obtenido de estas muestras es bajo. A pesar de esta gran desventaja, el uso de las nuevas TAAN como la detección de VPH mediante RPC en tiempo real hace posible detectar la infección aun cuando se dispone de bajas cantidades de $\mathrm{ADN}^{59}$.

La orina es una muestra no invasora y de fácil obtención lo que ha fomentado su uso, ya que esta produce un menor rechazo por parte de los pacientes en comparación al uso de exudados, los que deben ser recolectados mediante métodos invasores, produciendo incomodidad. Otra gran ventaja es que la muestra de orina puede ser recolectada por el propio paciente, lo que también minimiza su rechazo representando una gran ventaja al momento de compararla con otros tipos de muestras, disminuyendo ostensiblemente el costo del examen, ya que la mayoría de estas deben ser recolectadas por un especialista. Por esto, en la actualidad se están desarrollando técnicas de detección de ITS mediante el uso de orina y técnicas de amplificación de ácidos nucleicos como RPC multiplex, para lograr detectar más de una infección utilizando una única muestra de orina y un único test. Asimismo, este es un método más aceptado en la población masculina, en quienes la muestra de semen, necesaria en la actualidad para detectar ITSS, es obtenida por masturbación. Esto dificulta la detección oportuna y la concurrencia espontánea de estos pacientes a realizar este tipo de examen que les permita detectar una ITSS.

\section{Conclusión}

Las ITSS han sido catalogadas como un gran problema para la salud pública mundial, lo que está reflejado en el número de nuevos casos reportados, año a año. Estas infecciones silentes, son difíciles de estimar, ya que no presentan sintomatología y el paciente rara vez acude al médico por ellas, siendo detectadas muchas veces al consultar por otro problema médico como puede ser PAP alterados y/o infertilidad.

A través del tiempo, las técnicas utilizadas en la detección de ITSS han evolucionado, alcanzando altos porcentajes de sensibilidad y especificidad, lo que ha facilitado y mejorado su diagnóstico. Gran parte de estas técnicas utilizan muestras obtenidas por procedimientos invasores, siendo necesario un especialista para su recolección, lo que genera incomodidad a los pacientes y tratan de evitar el realizarse este tipo de prueba. Por esto surge la necesidad de buscar muestras obtenidas de manera menos invasora siendo una opción de ellas la orina, cuya toma de muestra no requiere de un especialista, puede ser recolectada por el propio paciente, representando una ventaja por sobre las muestras invasoras. La utilización de la muestra de orina para la detección de ITSS ayudaría a aumentar la pesquisa de pacientes que padecen de estas infecciones debido a la comodidad que proporciona la toma de este tipo de muestra clínica. Esto permitiría generar un catastro de pacientes infectados lo cual sería de gran utilidad en salud pública, pudiendo disminuir las tasas de infección a largo plazo y con esto reducir tanto la tasa de infertilidad y la morbi-mortalidad causada tanto por la presencia de virus oncológicos como inmunosupresores.

\section{Resumen}

La detección de infecciones de transmisión sexual silentes (ITSS) ha tomado gran relevancia, debido principalmente a su incremento en el mundo. Esto ha llevado a implementar diversas técnicas de laboratorio con la finalidad de la detección precoz de estas infecciones silentes para disminuir su incidencia. Las técnicas que habitualmente se utilizan para la detección e identificación de ITSS requieren de una muestra invasora (sangre, citobrush, entre otras), por lo que la orina podría ser una opción de muestra más simple y no invasora al momento que el paciente se deba someter a un examen para detectar alguna de estas infecciones. 


\section{Referencias bibliográficas}

1.- Santander C, Fich F, Salvo L. Normas de manejo y tratamiento de las infecciones de transmisión sexual (ITS). Primera parte. Rev Chilena Infectol 2012; 26: 174-90.

2.- WHO. Global incidence and prevalence of selected curable sexually transmitted infections - 2008. 2012.

3.- MINSAL. Informe Anual 2011 Gonorrea (CIE 10:A.54). 2011.

4.- Potterat J, Woodhouse D, Pratts C, Markewich G, Fogle J. Women contacts of men with gonorrhea: case-finding yields. Sex Transm Dis 1983; 10: 29-32.

5.- Sorrell I, White A, Pedersen A, Hails R, Boots $M$. The evolution of covert, silent infection as a parasite strategy. Proc Biol Sci 2009; 276: 221726.

6.- WHO/ICO. Information centre on HPV and cervical cancer (HPV information centre). Human papillomavirus and related cancers in Americas. Summary Report 2010. 2010.

7.- Bébéar C, de Barbeyrac B. Genital Chlamydia trachomatis infections. Clin Microbiol Infec 2009; 15: 4-10.

8.- Looker K, Garnett G, Schmid G. An estimate of the global prevalence and incidence of herpes simplex virus type 2 infection. Bull World Health Organ 2008; 86: 805-12.

9.- Rodríguez B, Santana F. Infecciones de transmisión sexual, calidad del semen e infertilidad. Rev Cubana Endocrinol 2008; 19 (3).

10.- Rivera R, Aguilera J, Larraín A. Epidemiología del virus papiloma humano (HPV). Rev Chil Obstet Ginecol 2002; 67: 501-6.

11.- Götz H, Lindbäck J, Ripa T, Arneborn M, Ramstedt K, Ekdahl K. Is the increase in notifications of Chlamydia trachomatis infections in Sweden the result of changes in prevalence, sampling frequency or diagnostic methods? Scand J Infect Dis 2002; 34: 28-34.

12.- Gupta S, Govindarajan S, Fong T, Redeker A. Spontaneous reactivation in chronic hepatitis B: patterns and natural history. J Clin Gastroenterol 1990; 12: 562-8.

13.- Lok A, Ward J, Perrillo R, McMahon B, Liang T. Reactivation of hepatitis B during immunosuppressive therapy: potentially fatal yet preventable. Ann Intern Med 2012; 156: 743-5.

14.- Vázquez F, Lepe J A, Otero L, Blanco M A, Aznar J. Diagnóstico microbiológico de las infecciones de transmisión sexual (2007). Enferm Infecc Microbiol Clin 2008; 26: 32-7.

15.- Black C, Morse S. The use of molecular techniques for the diagnosis and epidemiologic study of sexually transmitted infections. Curr Infect Dis Rep 2000; 2: 31-43.

16.- Aznar J, Blanco M, Lepe J, Otero L, Vázquez F. Diagnóstico microbiológico de las infecciones de transmisión sexual y otras infecciones genitales. Procedimientos en Microbiología Clínica de la SEIMC 2007; 24. https://www. seimc.org/contenidos/documentoscientificos/ procedimientosmicrobiologia/seimcprocedimientomicrobiologia24.pdf

17.- Vázquez F, Otero L, Ordás J, Junquera M, Varela J. Actualización en infecciones de transmisión sexual: epidemiología, diagnóstico y tratamiento. Enferm Infecc Microbiol Clin 2004; 22: 392-411.

18.- Pinilla G, Cubillos K, Rodríguez M. Bodas de plata de la reacción en cadena de la polimerasa (PCR). Nova 2008; 6: 65-75.

19.- Lee H. Ligase chain reaction. Biologicals 1996; 24: 197-9.

20.- Wroblewski J, Manhart L, Dickey K, Hudspeth $\mathrm{M}$, Totten P. Comparison of transcriptionmediated amplification and PCR assay results for various genital specimen types for detection of Mycoplasma genitalium. J Clin Microbiol 2006; 44: 3306-12.

21.- Edberg A, Jurstrand M, Johansson E, Wikander E, Hoog A, Ahlqvist T, et al. A comparative study of three different PCR assays for detection of Mycoplasma genitalium in urogenital specimens from men and women. J Med Microbiol 2008; 57: 304-9.

22.- Baczynska A, Svenstrup H, Fedder J, Birkelund S, Christiansen G. Development of real-time PCR for detection of Mycoplasma hominis. BMC Microbiol 2004; 4: 35.

23.- Cunningham S, Mandrekar J, Rosenblatt J, Patel R. Rapid PCR Detection of Mycoplasma hominis, Ureaplasma urealyticum, and Ureaplasma parvum. International J Bacteriol 2013; 2013: 7.

24.- Boga B, Grau M, Andreu L, Peinado M. Diagnóstico microbiológico de las infecciones por Mycoplasma spp. y Ureaplasma spp. Procedimientos en Microbiología Clínica de la SEIMC 2011; 40. https://www.seimc. org/contenidos/documentoscientificos/ procedimientosmicrobiologia/seimcprocedimientomicrobiologia40.pdf

25.- Alonso-Vega C, Wauters N, Vermeylen D, Muller M, Serruys E. A fatal case of Mycoplasma hominis meningoencephalitis in a full-term newborn. J Clin Microbiol 1997; 35: 286-7.

26.- Kuo C, Wang S, Wentworth B, Grayston J. Primary isolation of TRIC organisms in $\mathrm{HeLa}$ 229 cells treated with DEAE-dextran. J Infect Dis 1972; 125: 665-8.

27.- Rota T, Nichols R. Infection of cell cultures by trachoma agent: enhancement by DEAEdextran. J Infect Dis 1971; 124: 419-21.

28.- Stamm W, Tam M, Koester M, Cles L. Detection of Chlamydia trachomatis inclusions in McCoy cell cultures with fluoresceinconjugated monoclonal antibodies. J Clin Microbiol 1983; 17: 666-8.
29.- Parra-Sánchez M, Palomares J, Bernal S, González M, Sivianes N, Pérez L, et al. Evaluation of the cobas $4800 \mathrm{CT} / \mathrm{NG}$. Test for detecting Chlamydia trachomatis and Neisseria gonorrhoeae DNA in urogenital swabs and urine specimens. Diagn Microbiol Infec Dis 2012; 74: 338-42.

30.- Wisniewski C, White J, Michel C, MahilumTapay L, Magbanua J, Nadala E, et al. Optimal method of collection of first-void urine for diagnosis of Chlamydia trachomatis infection in men. J Clin Microbiol 2008; 46: 1466-9.

31.- CDC. Recommendations for the laboratorybased detection of Chlamydia trachomatis and Neisseria gonorrhoeae-2014. MMWR Recomm Rep 2014; 63: 1-19.

32.- Lawung R, Cherdtrakulkiat R, Prachayasittikul V. Protocol for a facile multiplex PCR for multi-antimicrobial resistance and gonococcus detection, in diagnosis of sexually transmitted diseases, C.R. MacKenzie and B. Henrich, editors. 2012, Humana Press. pag. 329-41.

33.- Dillner J. The serological response to papillomaviruses. Semin Cancer Biol 1999; 9: 423-30.

34.- Molijn A, Kleter B, Quint W, van Doorn L J. Molecular diagnosis of human papillomavirus (HPV) infections. J Clin Virol 2005; 32 Suppl 1: S43-51.

35.- Mateos M, Chacón de Antonio J, RodríguezDomínguez M, Sanz I, Rubio M. Evaluation of a prototype real-time PCR assay for the separate detection of human papilloma virus genotypes 16 and 18 and other high risk human papillomavirus in cervical cancer screening. Enferm Infecc Microbiol Clin 2011; 29: 411-4.

36.- Barzon L, Militello V, Pagni S, Palù G. Comparison of INNO-LiPA genotyping extra and hybrid capture 2 assays for detection of carcinogenic human papillomavirus genotypes. J Clin Virol 2012; 55: 256-61.

37.- Cañadas M, Cirigliano V, Darwich L, Sirera G, Coll J, Clotet B, et al. Comparison of the f-HPV typing ${ }^{\text {TM }}$ and Hybrid Capture II ${ }^{\circledR}$ assays for detection of high-risk HPV genotypes in cervical samples. J Virol Methods 2012; 183: 14-8

38.- Madhavan H, Priya K, Anand A, Therese K Detection of herpes simplex virus (HSV) genome using polymerase chain reaction (PCR) in clinical samples comparison of PCR with standard laboratory methods for the detection of HSV. J Clin Virol 1999; 14: 145-51.

39.- Heaton P, Espy M, Binnicker M. Evaluation of 2 multiplex real-time PCR assays for the detection of HSV-1/2 and varicella zoster virus directly from clinical samples. Diagn Microbiol Infect Dis 2015; 81: 169-70.

40.- Fleming D, McQuillan G, Johnson R, Nahmias A, Aral S, Lee F, et al. Herpes simplex virus type 2 in the United States, 1976 to 1994. N Engl J Med 1997; 337: 1105-11. 
41.- Strick L, Wald A. Diagnostics for herpes simplex virus: is PCR the new gold standard? Mol Diagn Ther 2006; 10: 17-28.

42.- Buelow D, Bankowski M, Fofana D, Gu Z, Pounds S, Hayden R. Comparison of two multiplexed PCR assays for the detection of HSV-1, HSV-2, and VZV with extracted and unextracted cutaneous and mucosal specimens. J Clin Virol 2013; 58: 84-8.

43.- Gitman M, Ferguson D, Landry M L. Comparison of Simplexa HSV 1 \& 2 PCR with culture, immunofluorescence, and laboratorydeveloped TaqMan PCR for detection of herpes simplex virus in swab specimens. J Clin Microbiol 2013; 51: 3765-9.

44.- García F, Álvarez M, Bernal C, Chueca N, Guillot V. Diagnóstico de laboratorio de la infección por el VIH, del tropismo viral y de las resistencias a los antirretrovirales. Enferm Infecc Microbiol Clin 2011; 29: 297-307.

45.- Buttò S, Suligoi B, Fanales-Belasio E, Raimondo M. Laboratory diagnostics for HIV infection. Ann Ist Super Sanita 2010; 46: 24-33.

46.- Weber B. Screening of HIV infection: role of molecular and immunological assays. Expert Rev Mol Diagn 2006; 6: 399-411.

47.- Cevenini R, Sarov I, Rumpianesi F, Donati M, Melega C, Varotti C, et al. Serum specific IgA antibody to Chlamydia trachomatis in patients with chlamydial infections detected by ELISA and an immunofluorescence test. J Clin Pathol 1984; 37: 686-91.

48.- Bosshard P. Usefulness of IgM-specific enzyme immunoassays for serodiagnosis of syphilis: comparative evaluation of three different assays. J Infect 2013; 67: 35-42.

49.- Isenberg H. Clinical Microbiology Procedures Handbook, 3rd Edition. 3 ed. American Society Mic Series. 2010; American Society for Microbiology.

50.- Martin-Hirsch P, Jarvis G, Kitchener H, Lilford R. Collection devices for obtaining cervical cytology samples. Cochrane Database Syst Rev 2000; 3: CD001036.

51.- Fernández D, Di Chiazza S, Veyretou F, González L, Romero M. Análisis de orina: estandarización y control de calidad. Acta Bioquímica Clínica Latinoamericana 2014; 48: 213-21.

52.- Comité de Microbiología Clínica, Sociedad Chilena de Infectología. Recomendaciones para el diagnóstico microbiológico de la infección urinaria. Rev Chilena Infectol 2001; 18: 57-63.

53.- Little P, Moore M, Turner S, Rumsby K, Warner G, Lowes J A, et al. Effectiveness of five different approaches in management of urinary tract infection: randomised controlled trial. BMJ 2010; 340: c199.

54.- Gayet-Ageron A, Ninet B, Toutous-Trellu L,
Lautenschlager S, Furrer H, Piguet V, et al. Assessment of a real-time PCR test to diagnose syphilis from diverse biological samples. Sex Transm Infect 2009; 85: 264-9.

55.- Lillis R, Nsuami M, Myers L, Martin D. Utility of urine, vaginal, cervical, and rectal specimens for detection of Mycoplasma genitalium in women. J Clin Microbiol 2011; 49: 1990-2.

56.- McKechnie M, Hillman R, Couldwell D, Kong F, Freedman E, Wang H, et al. Simultaneous identification of 14 genital microorganisms in urine by use of a multiplex PCR-based reverse line blot assay. J Clin Microbiol 2009; 47 : 1871-7.

57.- Silva R, Leon D, Viscarra T, Ili C, Roa J, Sánchez R, et al. Frequency of Chlamydia trachomatis infection in a group of women from Region of Araucania, Chile. Rev Chilena Infectol 2013; 30: 611-5.

58.- Sellors J, Lorincz A, Mahony J, Mielzynska I, Lytwyn A, Roth P, et al. Comparison of selfcollected vaginal, vulvar and urine samples with physician-collected cervical samples for human papillomavirus testing to detect high-grade squamous intraepithelial lesions. CMAJ 2000; 163: 513-8.

59.- Enerly E, Olofsson C, Nygård M. Monitoring human papillomavirus prevalence in urine samples: a review. Clin Epidemiol 2013; 5: 67-79. 